\title{
First-principles studies of lithium adsorption and diffusion on silicene with grain boundaries
}

Xiao Wang, ${ }^{1}$ Huazhong Liu, ${ }^{2}$ Marko Huttula, ${ }^{3}$ Youhua Luo, ${ }^{1}$ Meng Zhang, ${ }^{1,}$ Wei

$$
\mathrm{CaO}^{3, *}
$$

${ }^{1}$ Department of Physics, School of Science, East China University of Science and Technology, Shanghai 200237, China

${ }^{2}$ Department of Basic Courses, Wuhan Donghu University, Wuhan, 430212, China

${ }^{3}$ Nano and Molecular Systems Research Unit, University of Oulu, P.O. Box 3000, FIN-90014, Finland

Correspondence to: Meng Zhang (Email: mzhang@ecust.edu.cn) and Wei Cao (Email: wei.cao@oulu.fi) 


\begin{abstract}
As a close relative to graphene, silicene is advanced in high lithium capacity, yet attracting various manipulation strategies to promote its role in energy storage. Following grain boundary (GB) engineering route as widely used in graphene studies, in this work, first-principles calculations were performed to investigate adsorption and diffusion behaviors of lithium on silicene with GBs of $4 \mid 8$ or $5|5| 8$ defects. In both GB forms, donation of the $\mathrm{Li} 2 \mathrm{~s}$ electron to the GBs significantly increases the $\mathrm{Li}$ adsorption energy, while small energy barriers facilitate the Li migration on the silicene surface. Furthermore, the large hole of GB (4-8) also permits easy penetration of the Li ions through the defective silicene sieve. These important features demonstrate GBs are beneficial for enhancing capacity and charge speed of the $\mathrm{Li}$ batteries. Thus, superior anodes made of silicene with GBs are expected to serve a key solution for future energy storages.
\end{abstract}




\section{INTRODUCTION}

The rechargeable lithium ion batteries (LIBs) are the predominant energy reservoirs in electric vehicles and portable electronics. However, future batteries require smaller LIB dimensions, lighter weights, along with higher energy densities. To meet these stringent requirements, extensive researches have been emphasized on explorations of the advanced electrode materials which can provide high charge capacity, long cyclic stability, high-rate capability and safety. ${ }^{[1-3]}$ Graphene and oxidized graphene have been found with higher capacities of 600 to $1000 \mathrm{mAh} / \mathrm{g}^{[4,5]}$ than the bulk counterpart of graphite $(372 \mathrm{mAh} / \mathrm{g}) .{ }^{[6]} \mathrm{In}$ analogy to these organic monolayers, other two-dimensional (2D) materials were also studied and considered as important candidates for the LIBs anodes. So far, explorations have been searched within naturally occurring species as graphdiyne, ${ }^{[7]}$ molybdenum disulfide $\left(\mathrm{MoS}_{2}\right)^{[8]}$ and boron carbon nitride nanosheets, ${ }^{[9]}$ etc.

The synthetic monolayers may also act as anode materials for Li storage. Recently, silicene - the silicon monolayer, has been synthesized on various metal substrates such as $\mathrm{Ag}$, Ir and $\mathrm{ZrB}_{2} \cdot{ }^{[10-13]}$ Preliminary results have shown that high Li capacity is feasible on nanostructured silicon (e.g. silicon nanowires, ${ }^{[14,15]}$ nanoparticles ${ }^{[16]}$ ) but with small volume deformations of the matrices. Thus, silicene is also expected to be a good candidate for LIB electrodes because of its nature of low-dimensional silicon and structural similarity to graphene. In fact, theoretical studies have indicated that the binding energy between $\mathrm{Li}$ and silicene is $2.2 \mathrm{eV}$ per $\mathrm{Li}$ atom and the barriers for Li diffusion are less than $0.6 \mathrm{eV},{ }^{[17,18]}$ much lower than that 
of Li diffusion on graphene. ${ }^{[19,20]}$ Moreover, silicene is exempt from irreversible structural changes during lithiation/delithiation processes in comparison with crystalline silicon. ${ }^{[21]}$ Thus, silicene may act as an alternative of graphene on the LIB electrodes.

However, the unavoidable defects associated with material synthesis bring substantial impacts, either beneficial or detrimental, on the physical, chemical and electronic properties of the low dimensional materials. ${ }^{[22,23]}$ Several works have been concentrated on the structural and electronic properties of point defects in free-standing silicene. ${ }^{[24-27]}$ Another important kind of structurally robust defect is topological line defects (LDs), which remain stable against local geometrical rearrangements. ${ }^{[28,29]}$ Different types of line defects are already found at the grain boundaries (GBs) of graphene, ${ }^{[30,31]}$ hexagonal boron nitride, ${ }^{[32]}$ molybdenum disulphide, ${ }^{[33]}$ etc. As for silicene, several types of GBs have been identified during the growth on $\operatorname{Ag}(111)$ via molecular-beam epitaxy technique. ${ }^{[26]}$ A large number of defect clusters were also observed in the scanning tunneling microscope (STM) images of silicene, where more than three Si vacancies compose the extended line defects. ${ }^{[34]}$ Moreover, the STM result showed that patterned defect structures behave as vacancies, pentagon-heptagon pairs, square-octagon pairs on single-layer silica films on $\mathrm{Ru}(0001) .{ }^{[35]}$ Furthermore, in the graphene studies, introducing GBs will enhance the capacity of $\mathrm{Li}$ (or $\mathrm{Na}$ ) adatoms and lower energy barriers of the diffusions of these adatoms along the GBs. Thus, graphene with GBs are considered beneficial for the LIBs or sodium ion batteries (NIBs). ${ }^{[36,37]}$ 
Despite dedications in materials synthesis and property studies, the role of GBs remains elusive for the adsorption and diffusion of lithium on silicene. A key question arises that if such GB defects will be beneficial as the case of graphene, or introduce shrinkage of the battery capacity. In this work, we present a systematic computational investigation of the adsorption behavior of $\mathrm{Li}$ on silicene with GBs. The surface diffusion and diffusion through silicene were also studied to evaluate the energy barriers that affect the charging rates and cyclic performance. To the best of our knowledge, it is the first time to apply the GB engineering for silicene in energy researches.

\section{COMPUTATIONAL METHOD}

The computations are carried out using unrestricted spin-polarized DFT, implemented in the Dmol3 code. ${ }^{[38-40]}$ A PerdewBurkeErnzerhof (PBE) functional of generalized-gradient approximation (GGA) is used for calculating exchange correlation functions. ${ }^{[41]}$ The double numerical plus polarization function (DNP) is chosen as the atomic orbital basis set. ${ }^{[40]}$ The dispersion corrections of Tkatchenko-Scheffler ${ }^{[42]}$ are adopted to describe the van der Waals interactions in our systems. A k-points grid sampling equivalent ${ }^{[43]} 10 \times 10 \times 1$ in the unitary cell is considered. A basis set cutoff of $4.6 \AA$ is employed for all systems, and the effect of periodic boundaries is negligible. All atoms are allowed to relax until the forces on each atom are smaller than $0.001 \mathrm{Ha} / \AA$, with the convergence threshold energy of 
$10^{-6} \mathrm{Ha}$.

Two kinds of grain boundaries are considered. The first one is the $4 \mid 8$ defect along the armchair boundary $(\mathrm{GB}(4-8))$ and the second the $5|5| 8$ defect along the zigzag chain $(\mathrm{GB}(5-8))$. We simulated $2 \mathrm{D}$ sheets by employing periodic boundary conditions. A vacuum of $20 \AA$ is used to avoid the interactions between periodic images. The relative stability of the LD defect comparing with freestanding silicene can be estimated by the formation energy, $\mathrm{E}_{f}$, which is defined as

$$
\mathrm{E}_{f}=\left[\mathrm{E}_{G B}-\mathrm{E}_{P}\right] / \mathrm{L}
$$

where $\mathrm{E}_{G B}$ and $\mathrm{E}_{P}$ are the total energies of the defective and pristine silicene, respectively, and L represents length of the grain boundary.

Four typical adsorption sites are considered as the possible adsorption sites on pristine silicene, including the hollow $(\mathrm{H})$ site above the center of a hexagon ring, the site on the top of Si atom in higher plane (T), the site on the top of the $\mathrm{Si}$ atom in lower plane (V) and the bridge (B) site above the midpoint of a Si-Si bond. Based on our previous results of Li adsorption on non-defective matrix, ${ }^{[21]}$ we considered two types of adsorption sites on the defected silicene: the $\mathrm{H}$ site above the center of a polygon (four-membered, pentagon, or octagon) ring and $\mathrm{T}$ site on the top of $\mathrm{Si}$ atoms in higher plane around the defective region. The adsorption energy ( $\left.\mathrm{E}_{\mathrm{ads}}\right)$ of $\mathrm{Li}$ is obtained as

$$
\mathrm{E}_{\mathrm{ads}}=\mathrm{E}(\text { total })-\mathrm{E}(\text { sheet })-\mathrm{E}(\mathrm{Li})
$$

where $\mathrm{E}($ total), $\mathrm{E}$ (sheet), and $\mathrm{E}(\mathrm{Li})$ are the total energies of relaxed silicene with $\mathrm{Li}$ 
adsorption, the isolated silicene sheet and the isolated Li atom. According to the definition, negative adsorption energy denotes an energetically favorable $\mathrm{Li}$ adsorption to silicene.

To study the effect of grain boundaries on the diffusion of $\mathrm{Li}$ on silicene, the energy barrier $\Delta \mathrm{E}$ of Li diffusion is calculated. Linear synchronous transit (LST) with the quadratic synchronous transit (QST) method was used to search for minimum energy path. ${ }^{[44]}$ Then the transition state was confirmed by the nudged elastic band (NEB) method. ${ }^{[45]}$

\section{RESULTS AND DISCUSSIONS}

\subsection{GBs in silicene monolayer}

The GB(4-8) in silicene is formed along the armchair edges as shown in Figure 1. After full relaxation, the neighboring half-lattices couple to each other and form periodic arrays of octagonal and four-membered rings. The GB(5-8) contains two pentagons spatially separated by an octagonal ring, following the removal of silicon dimmer stilted $30^{\circ}$ from the zigzag chain. The formation energies for $\mathrm{GB}(4-8)$ and $\mathrm{GB}(5-8)$ structures are $0.20 \mathrm{eV} / \AA$ and $0.15 \mathrm{eV} / \AA$, respectively. These results suggest that the formation of GBs in a silicene sheet is easier compared with its counterpart in graphene. And the $\mathrm{GB}(5-8)$ is more energetically favorable than $\mathrm{GB}(4-8)$, in agreement with previous calculation. ${ }^{[46]}$ 
(a)

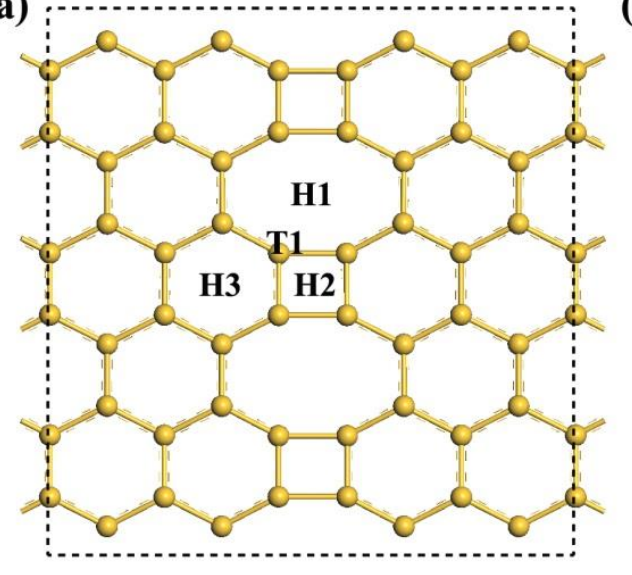

(b)

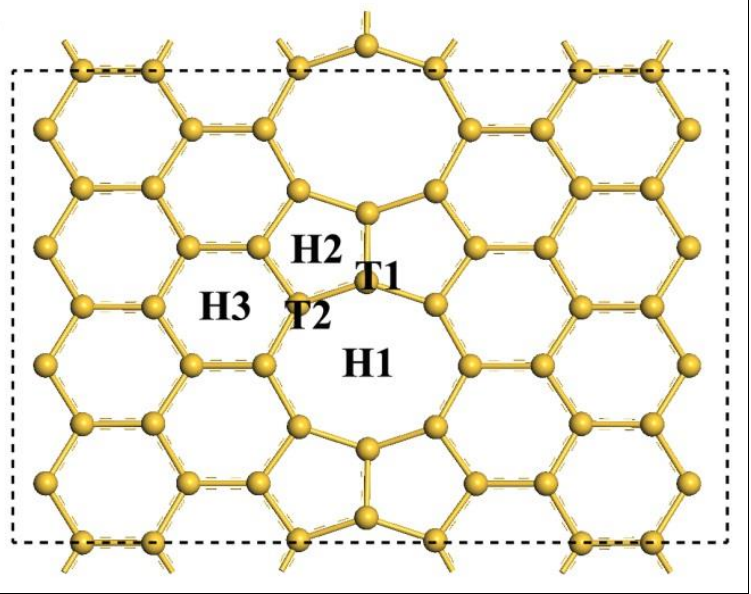

FIGURE 1 Optimized atomic structures of silicene with (a) $4 \mid 8$ and (b) $5|5| 8$ GBs.

The considered sites for adsorption of a Li atom are indicated by the black labels.

\subsection{Adsorption of Li on silicene monolayer with GBs}

In the case of Li adsorption on perfect silicene, a hollow site with one $\mathrm{Li}$ ion is the lowest energy configuration with adsorption energy of $-2.41 \mathrm{eV}$ according to our previous study. ${ }^{[21]}$ The metal adatom drift over to an $\mathrm{H}$ site when placed above a B site.

For Li adsorption on $\mathrm{GB}(4-8)$ sheet, considering the symmetry, various adsorption sites are examined including Li adsorption on the hollow site of polygonal rings and at the top of Si atoms round the defective region as shown in Figure 1. All the adsorption energies of stable configurations are summarized in Table 1. It is found that the most stable site for $\mathrm{Li}$ adsorption is the hollow site of the octagonal ring $(\mathrm{H} 1)$ (Figure 2). The $\mathrm{E}_{\text {ads }}$ is $0.47 \mathrm{eV}$ lower than the adsorption energy on the hollow site of perfect silicene. The distance $\left(\mathrm{d}_{\mathrm{Li}-\mathrm{Si}}\right)$ of the adsorbed Li atom and silicene surface is 
$0.912 \AA$, which is also much shorter than the non-defective case due to its larger adsorption energy. In addition, for Li adsorbed on different sites above GB(4-8), the stability of Li adatom from high to low exhibits the following order: octagonal ring > hexagonal ring $>$ four-membered ring $>$ the top of Si atom.

TABLE 1 The stable configurations of Li adsorption on defective silicene sheets and their adsorption energies ( $\left.E_{\text {ads }}\right)$.

\begin{tabular}{cccc}
\hline Li adsorption on $\mathrm{GB}(4-8)$ & \multicolumn{2}{l}{$\mathrm{Li}$ adsorption on $\mathrm{GB}(5-8)$} \\
\hline Model & $\mathrm{E}_{\text {ads }}(\mathrm{eV})$ & Model & $\mathrm{E}_{\text {ads }}(\mathrm{eV})$ \\
\hline $\mathrm{H} 1$ & -2.88 & $\mathrm{H} 1$ & -2.74 \\
$\mathrm{H} 2$ & -2.61 & $\mathrm{H} 2$ & -3.09 \\
\hline $\mathrm{H} 3$ & -2.81 & $\mathrm{H} 3$ & -2.85 \\
\hline $\mathrm{T} 1$ & -2.58 & $\mathrm{~T} 1$ & -2.88 \\
\hline
\end{tabular}




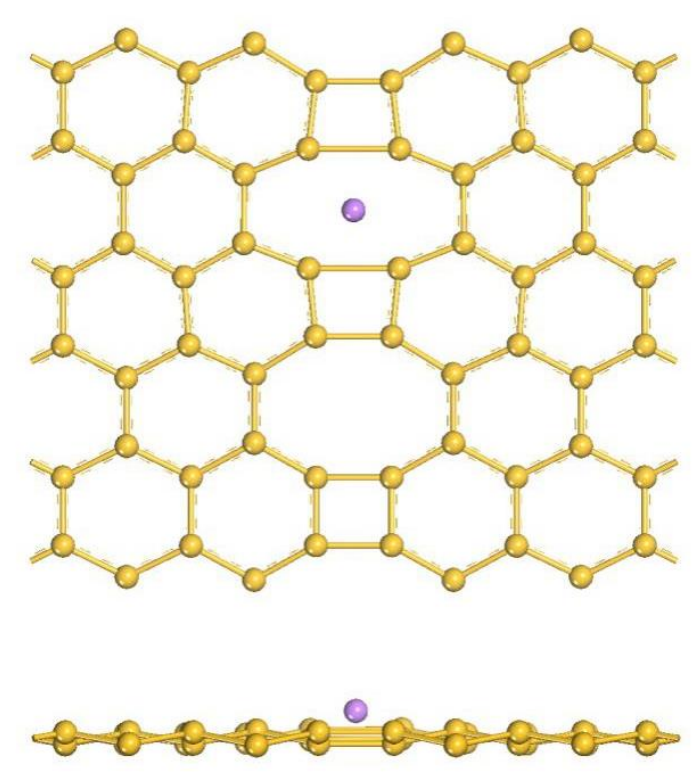

FIGURE 2 Optimized structure for Li adsorbed in the plane of an octagonal ring of GB(4-8): (a) top view and (b) side view.

For the GB(5-8) structure, various adsorption sites were considered as shown in Figure 1. It is found that the $\mathrm{H} 2$ site (see Table 1 and Figure 3 ) is the most stable one for $\mathrm{Li}$ adsorption with an $\mathrm{E}_{\text {ads }}$ of $-3.09 \mathrm{eV}$. For the different hollow sites above the polygonal rings of $\mathrm{GB}(5-8)$, the stability of a $\mathrm{Li}$ adatom from high to low exhibits the following order: pentagonal ring $>$ hexagonal ring $>$ octagonal ring. It is reversed as compared with the case of $\mathrm{GB}(4-8)$, in which octagonal ring is the most stable site for $\mathrm{Li}$ adsorption, suggesting the adsorption of $\mathrm{Li}$ in silicene depends on the kinds of grain boundaries. In addition, $\mathrm{Li}$ adsorbed at the top of $\mathrm{Si}$ atoms exhibits higher stability than on octagonal ring. Similar to the case of $\mathrm{GB}(4-8)$, Li adatom energetically prefers the sites around the defect region to those at non-defective region. The interaction of $\mathrm{Li}$ adatom and $\mathrm{GB}(5-8)$ is stronger than that of Li adatom and 
GB(4-8). Negative adsorption energies for a range of adsorption sites indicate that $\mathrm{Li}$ storage over silicene with GBs is a spontaneous process. Generally speaking, Li clusters may form with increasing the Li content. The formation of Li cluster would reduce the charge ability of LIBs and induce dendrite growth of lithium, which is harmful to battery safeties. Thus, we also investigated Li dimer adsorption on the defective systems. A Li dimer is firstly placed on silicene assumed that the hollow site is energetically favorable for each $\mathrm{Li}$ atoms and both $\mathrm{Li}$ atoms contact with the silicene surface. After optimization, structural changes can be clearly observed in Figure 4. The bond length of Li dimer is largely increased and the dimer cannot keep its configuration. Finally, two Li atoms from the dimer are separately adsorbed on the different hollow centers of $\mathrm{GB}(4-8)$ or $\mathrm{GB}(5-8)$ with average adsorption energies of $-2.77 \mathrm{eV} /$ atom or $-2.74 \mathrm{eV} /$ atom. The absolute values of these energies are smaller than those from single Li atom adsorption cases as shown in Table 1, but much larger than the cohesive energy of bulk bcc lithium $\left(-1.68\right.$ eV/atom) ${ }^{[47,48]}$ The results indicate that the existence of GBs could prevent the formation of Li clusters on silicene and suggest that Li atoms prefer adsorption on silicene with GBs. 


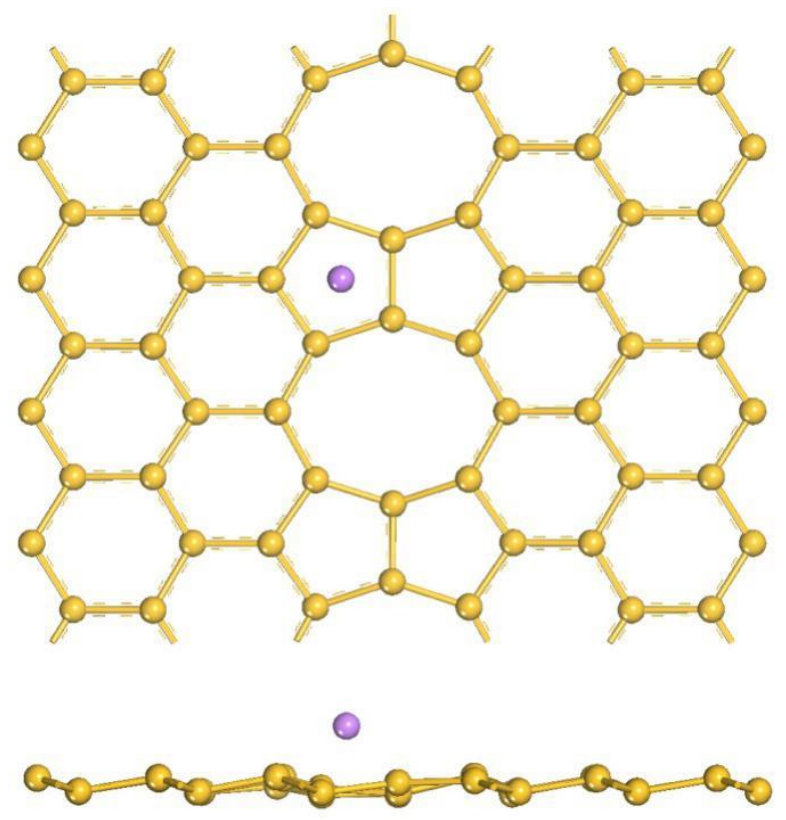

FIGURE 3 Optimized structure for Li adsorbed at the top of $\mathrm{H} 2$ site of GB(5-8): (a) top view and (b) side view.

(a)

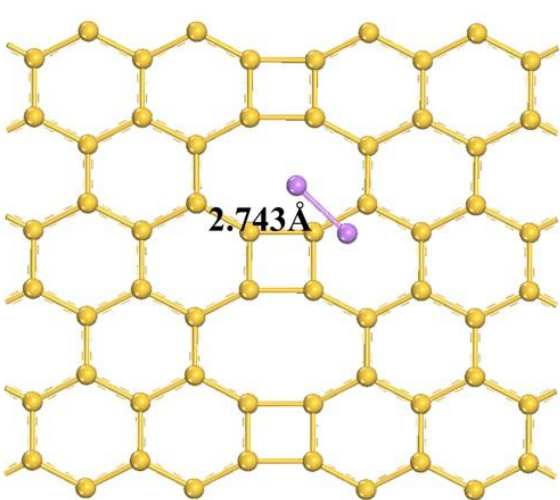

(c)

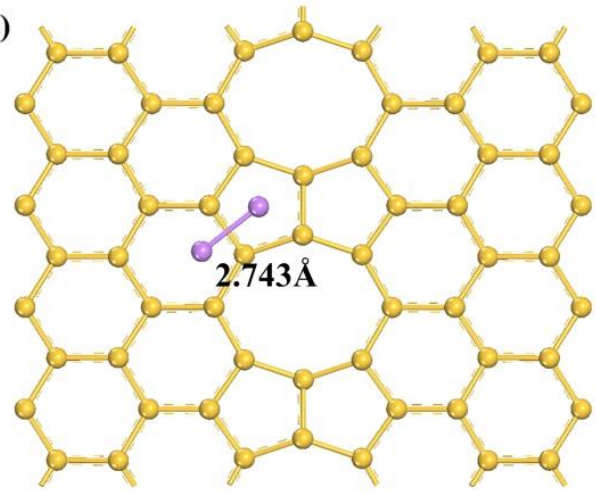

(b)

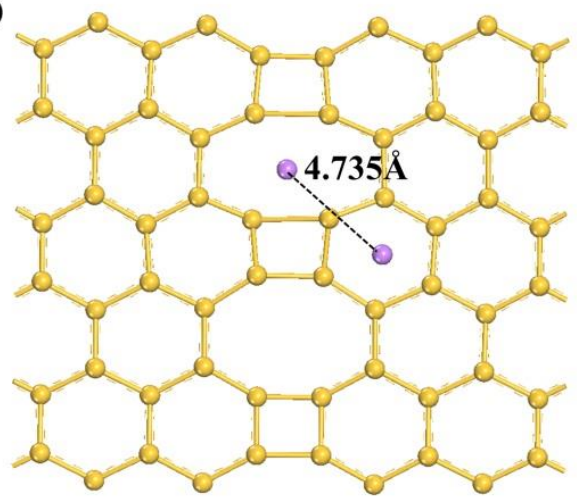

(d)

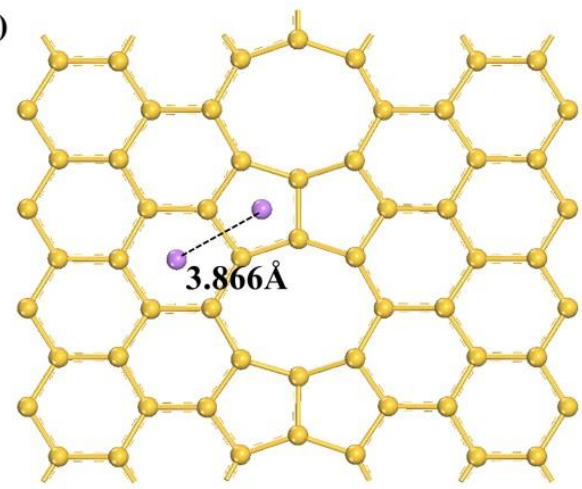

FIGURE 4 (a) and (c) are configurations that Li dimer adsorbs on GB(4-8) or GB (5-8) before optimization, (b) and (d) are that after optimization. 


\subsection{Electronic properties of silicene with $\mathrm{Li}$ adsorption}

To analyze the nature of Li-silicene interaction, we compute the electronic structures of silicene with the adsorbed Li. The projected density of states (PDOS) of the GB(4-8) with Li adsorption in Figure 5 show that after Li adsorption, the valence states of the system consist mainly of $3 p$ states of $\mathrm{Si}$ atoms and $2 \mathrm{p}$ states of Li. The strong hybridization between the $\mathrm{p}$ orbitals of $\mathrm{Li}$ and the four $\mathrm{Si}$ atoms denotes the typical feature of chemical adsorption. Figure 6 presents the deformation charge density of this system. Obviously, strong combined ionic and covalent Li-Si bonds are formed after adsorption as reflected in the length of the $\mathrm{Si}-\mathrm{Si}$ bonds around the $\mathrm{Li}$ atom elongating about $20 \%$ and consequently swelling the octagonal Si ring. The charge accumulation takes place near the puckered octagonal ring of $\mathrm{GB}(4-8)$ while charge-depleted regions surround the Li atom, indicating net charge transfer from the metal adatom to silicene. The Hirsheld charge analysis results further show that the charge transfer mainly occurs between the nearest up- and down-Si atoms in octagonal ring and $\mathrm{Li}$ atom. With the charge transferring, $\mathrm{Li}$ atom injects 0.12 e into the antibonding orbitals of these reactive Si atoms. The strong hybridization between $\mathrm{Li}$ and $\mathrm{Si}$ as well as the notable charge transfer verify that the strong $\mathrm{Si}-\mathrm{Li}$ interaction exists in silicene with GBs and results in the high stability of the structure. 


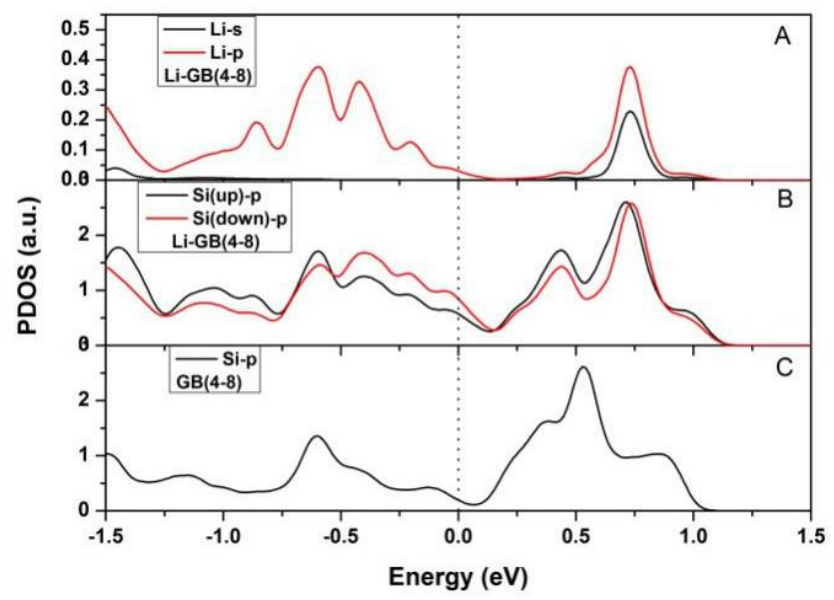

FIGURE 5 Density of states for the GB(4-8) with and without Li adsorption. A and B are the PDOS of the adatom $\mathrm{Li}$ and the defective region of $\mathrm{Si}$ atoms in $\mathrm{Li}$ adsorbed GB(4-8). Si(up) represent the nearest $\mathrm{Si}$ atoms above $\mathrm{Li}$ atom in Figure 2, and $\mathrm{Si}($ down) mean the nearest $\mathrm{Si}$ atoms below $\mathrm{Li}$ atom. For comparison, $\mathrm{C}$ is the DOS of the $\mathrm{Si}$ atoms in GB site in $\mathrm{GB}(4-8)$ without $\mathrm{Li}$ adsorption.

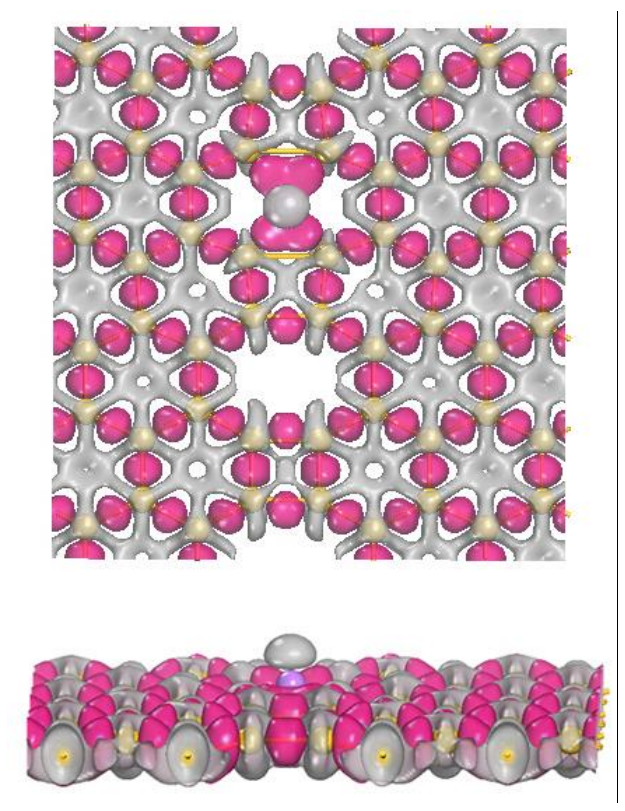

FIGURE 6 Top view and side view of deformation charge density plots for the most stable adsorption sites for Li over GB(4-8). Red and grey regions indicate charge accumulation and depletion, respectively. 
The bonding mechanism of $\mathrm{Li}$ atom adsorption on $\mathrm{GB}(5-8)$ is also presented in Figure 7. Detailed studies of DOS show that the $p$ states of Li atoms hybridize with $p$ states of Si atom in pentagon ring with a wide broadening which illustrates that the interactions between $\mathrm{Li}$ atom and $\mathrm{GB}(5-8)$ is also a strong chemical adsorption, similar to Li with $\mathrm{GB}(4-8)$. The wider energy states broadening suggests a stronger hybridization between pentagon ring and Li atom. According to Hirsheld analysis, Li atom transfers 0.25 e into $\mathrm{GB}(5-8)$. The larger charge transfer shows a stronger adsorption, in agreement with the PDOS analysis. However, with the electrons transfer into the bonding orbitals of pentagon ring, the Si-Si bonds of pentagon ring is subjected to very small changes (with the elongation of about $\sim 1 \%$ ), implying the stability of pentagon ring defect is larger than the octagonal ring defect. This is reasonable since the octagonal ring has a more loose geometric structure than the pentagon ring. It is also worth noting that line defects can significantly modify the electronic properties of silicene which transform semimetallic silicene into metallic. After Li adsorption, the metallic characteristic of both $\mathrm{GB}(4-8)$ and $\mathrm{GB}(5-8)$ remain. 


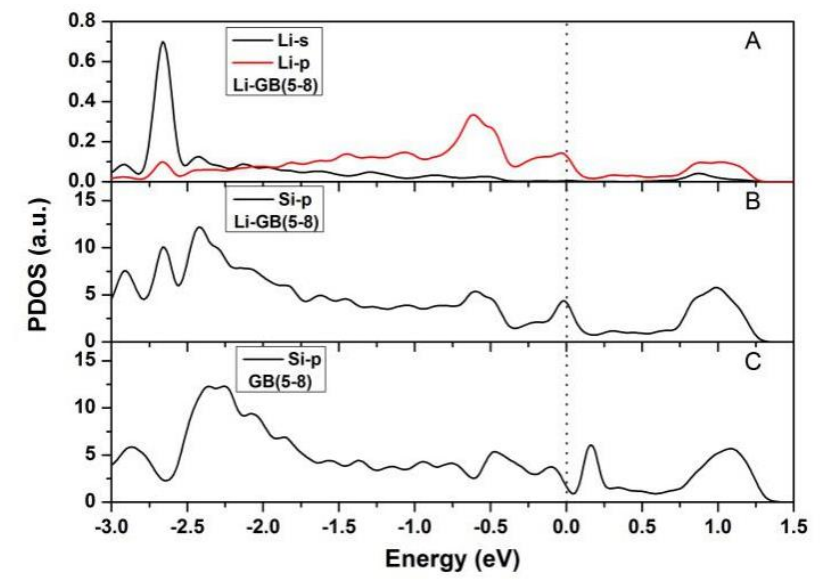

FIGURE 7 Density of states for the GB(5-8) with and without Li adsorption. A and B are the PDOS of the adatom $\mathrm{Li}$ and the defective region of $\mathrm{Si}$ atoms in $\mathrm{Li}$ adsorbed $\mathrm{GB}(5-8)$. For comparison, $\mathrm{C}$ is the DOS of the $\mathrm{Si}$ atoms in GB site in $\mathrm{GB}(5-8)$ without Li adsorption.

\subsection{Diffusion of a Li adatom on silicene}

As the charging rate of the LIBs relies on the Li ion mobility in the anode material, we further studied the Li diffusion on the silicene monolayer with GBs. When $\mathrm{Li}$ adatom diffuses on a perfect silicene, it is known that the Li atom would move from a hollow site to a nearest neighboring one by passing the top of $\mathrm{Si}$ atoms with an energy barrier of about $0.27 \mathrm{eV}$. For the diffusion of a $\mathrm{Li}$ atom on the silicene with GBs, we consider two types of diffusion paths, namely, the migration along and perpendicular to the GB. The calculated energy profiles for the $\mathrm{Li}$ atom diffusion between two neighboring sites on the $\mathrm{GB}(4-8)(\mathrm{H} 1$ to $\mathrm{H} 2$ and $\mathrm{H} 1$ to $\mathrm{H} 3$ ) are indicated in Figure 8 and the detailed results are listed in Table 2. Similar to the diffusion on the pristine matrix, the paths between two hollow sites are curves bent 
towards a silicon atom. $0.26 \mathrm{eV}$ is needed for lithium to diffuse out of the octagonal ring. Although the Li adatom may be trapped at the hollow site above the octagonal ring of $\mathrm{GB}(4-8)$ with larger adsorption energy, the trap will not obviously influence the diffusion energy of $\mathrm{Li}$ on the surface of silicene. Then the energy barrier for $\mathrm{Li}$ moving into the four-membered ring or the hexagonal ring is $0.03 \mathrm{eV}$ or $0.08 \mathrm{eV}$, respectively, suggesting a tendency of Li diffusion along the boundary. In addition, the energy barriers for the forward diffusion of a Li adatom are larger than those of the backward diffusion. As a result, the Li adatom tends to diffuse toward the octagonal ring site along the boundary.

Two diffusion paths were considered for the Li diffusion on $\mathrm{GB}(5-8)$ (H2 to H1 and $\mathrm{H} 2$ to $\mathrm{H} 3$ ), and the energy curves as a function of relative diffusion coordinates are shown in Figure 9. The energy barrier is $0.19 \mathrm{eV}$ and $0.22 \mathrm{eV}$ for Li diffusing from position $\mathrm{H} 2$ to $\mathrm{T} 1$ and $\mathrm{T} 2$, respectively. Compared with $\mathrm{Li}$ diffusion on the $\mathrm{GB}(4-8)$, Li adatom prefers to diffuse perpendicular to boundary in $\mathrm{GB}(5-8)$. The total energy barrier of Li moving from $\mathrm{H} 2$ to $\mathrm{H} 3$ decreases about $0.14 \mathrm{eV}$ compared with the value of Li diffusion along the boundary. It is noted from Table 2 that the diffusion barriers for backward diffusion were largely reduced, therefore, the Li atom also tends to diffuse to the defective pentagon. The maximum diffusion barriers are 0.16 and $0.10 \mathrm{eV}$ for backward diffusion of $\mathrm{Li}$ atoms from $\mathrm{H} 1$ or $\mathrm{H} 3$ site to $\mathrm{H} 2$, respectively. From these results, we can conclude that the GBs not only enhance the adsorption of $\mathrm{Li}$, but also affect the Li diffusion behavior on silicene, especially for the backward diffusion. 

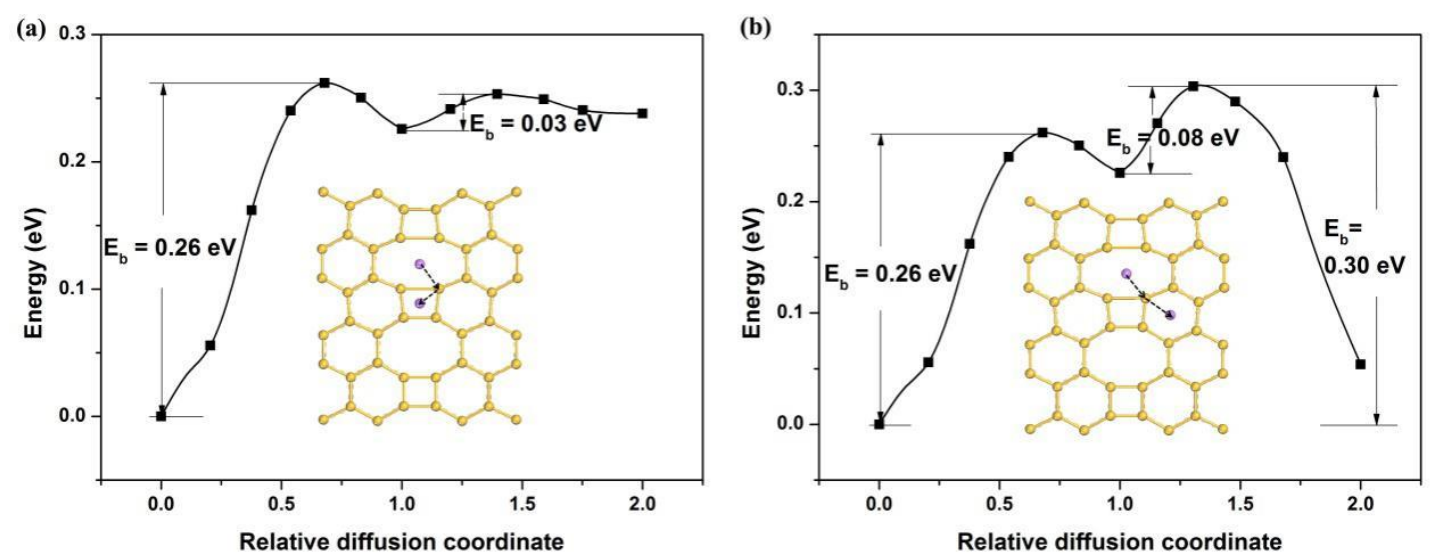

FIGURE 8 Energy curves for Li ion diffusion on silicene with $4 \mid 8$ GB following the path of (a) H1 to $\mathrm{H} 2$ and (b) $\mathrm{H} 1$ to $\mathrm{H} 3$.
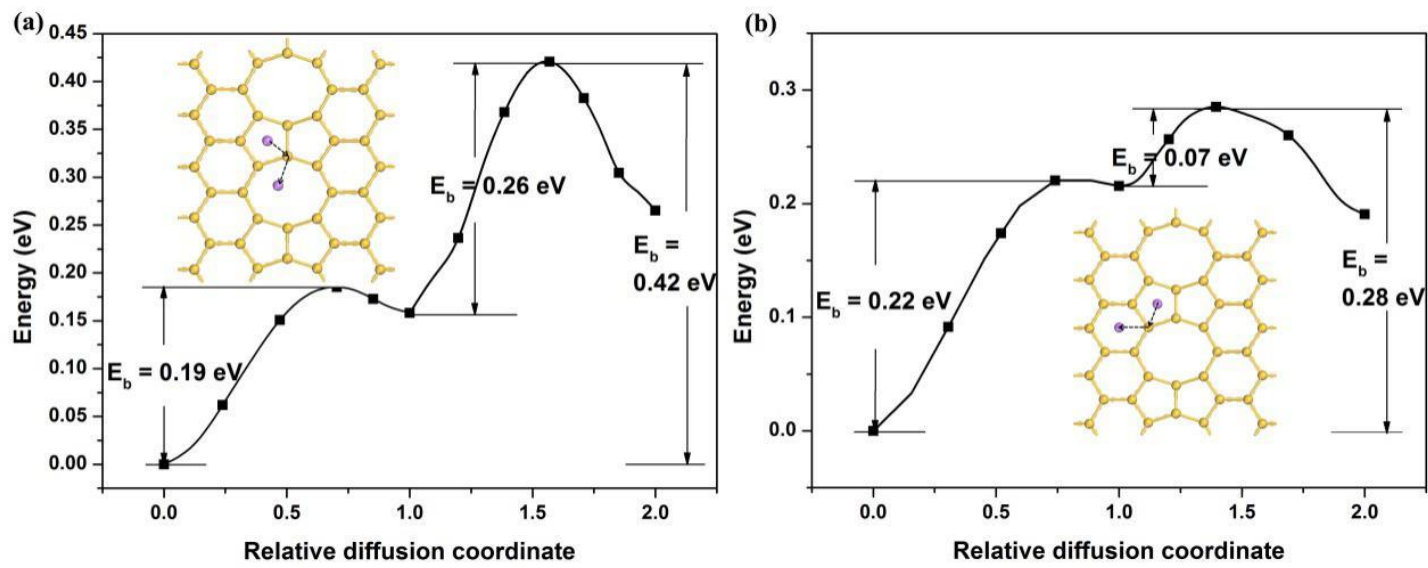

FIGURE 9 Energy curves for Li ion diffusion on silicene with $5|5| 8 \mathrm{~GB}$ following the path of (a) H2 to $\mathrm{H} 1$ and (b) $\mathrm{H} 2$ to $\mathrm{H} 3$. 
TABLE 2 Energy barriers $\left(E_{b}\right.$, in $\left.\mathrm{eV}\right)$ for the diffusion of $\mathrm{Li}$ on silicene with $4 \mid 8$ and $5|5| 8$ GBs.

\begin{tabular}{cccccc}
\hline System & along boundary & perpendicular to boundary & Through the \\
& & & & plane \\
\hline $\mathrm{GB}(4-8)$ & $\mathrm{H} 1 \rightarrow \mathrm{T} 1$ & $\mathrm{~T} 1 \rightarrow \mathrm{H} 2$ & $\mathrm{H} 1 \rightarrow \mathrm{T} 1$ & $\mathrm{~T} 1 \rightarrow \mathrm{H} 3$ & octagon \\
\hline $\mathrm{E}_{\mathrm{b}(\mathrm{eV})}$ & $0.26(0.04)$ & $0.03(0.02)$ & $0.26(0.04)$ & $0.08(0.25)$ & 0.04 \\
\hline $\mathrm{GB}(5-8)$ & $\mathrm{H} 2 \rightarrow \mathrm{T} 1$ & $\mathrm{~T} 1 \rightarrow \mathrm{H} 1$ & $\mathrm{H} 2 \rightarrow \mathrm{T} 2$ & $\mathrm{~T} 2 \rightarrow \mathrm{H} 3$ & pentagon \\
\hline $\mathrm{E}_{\mathrm{b}}(\mathrm{eV})$ & $0.19(0.03)$ & $0.26(0.16)$ & $0.22(0.01)$ & $0.07(0.10)$ & 2.92 \\
\hline
\end{tabular}

*The values in the parentheses correspond to the energy barrier of a backward diffusion.

\subsection{Migration of Li through silicene monolayer with GBs}

The diffusion of $\mathrm{Li}$ through the defects is also worthy exploring because $2 \mathrm{D}$ materials would likely be stacked in layers as the surface-to-volume ratio is higher than having only a single sheet. ${ }^{[49,50]}$ Additionally, ion orientations on double-sided monolayers show exceptional properties due to geometrical arrangements. ${ }^{[51]}$ Previous studies have shown that it is difficult for $\mathrm{Li}$ ions to get through from one side of graphene to the other side because of the large energy barriers. ${ }^{[19,52]}$ To compare silicene with graphene, we investigated the diffusion barriers through the sheet and our value for pristine silicene is $1.37 \mathrm{eV}$, which is much smaller than that of $\mathrm{Li}$ 
through the graphene. This is because the area of the hollow site of graphene is smaller than that of silicene. The larger area for silicene makes it easier for lithium to get through. Table 2 and Figure 10 clearly denote free diffusion of Li from one side to another side of the silicene plane with the $4 \mid 8 \mathrm{~GB}$. As a result of the hollow structure, diffusing through the center of octagonal ring requires only $0.04 \mathrm{eV}$, which suggests lithium atoms can pass through the hollow site with a high probability.

The mechanism of Li penetration to the defective silicene is also studied. When $\mathrm{Li}$ adsorbs on silicene, it possesses a positive charge after donating $2 \mathrm{~s}$ electron. The extra charge transferred from Li mostly stays at $\mathrm{Si}$ atoms near the $\mathrm{Li}$ atom, hence, the interactions between them can be ascribed to the coulomb interaction. The octagonal ring in $\mathrm{GB}(4-8)$ provides a large open space for Li penetration. This gives electrostatic charge overlapping minimized and a large bond length of $2.552 \AA$ at the barrier state could be found as shown in Figure 11(a), larger than the bond length of $2.369 \AA$ for the Li-Si dimer. Thus, steric hindrance is minimized. The charge difference between the adsorption and the barrier states is 0.03 e. These factors induced a very small diffusion barrier height $0.04 \mathrm{eV}$.

Since the Li atom prefers to adsorb on the defective pentagon in the case of $\mathrm{GB}(5-8)$, it is also worthy to investigate diffusion behavior of Li through the hole. However, a penetration energy of $2.92 \mathrm{eV}$ was calculated, even larger than the value of the Li diffusion through the hexagonal hole in a perfect silicene. As the Li approaches to the barrier site, the available space for $\mathrm{Li}$ is narrow with a short separation distance of $1.903 \AA$, invoking severe charge overlapping between $\mathrm{Li}$ and 
adjacent silicon atoms. This increases repulsive forces, giving rise to large diffusion barrier height. Thus, among the studied cases, the GB of $4 \mid 8$ serves best diffusion channels for the Li via diffusions through the octagonal ring and then freely go through it to another surface or the surface of other silicene layer for silicene-based nanostructures.

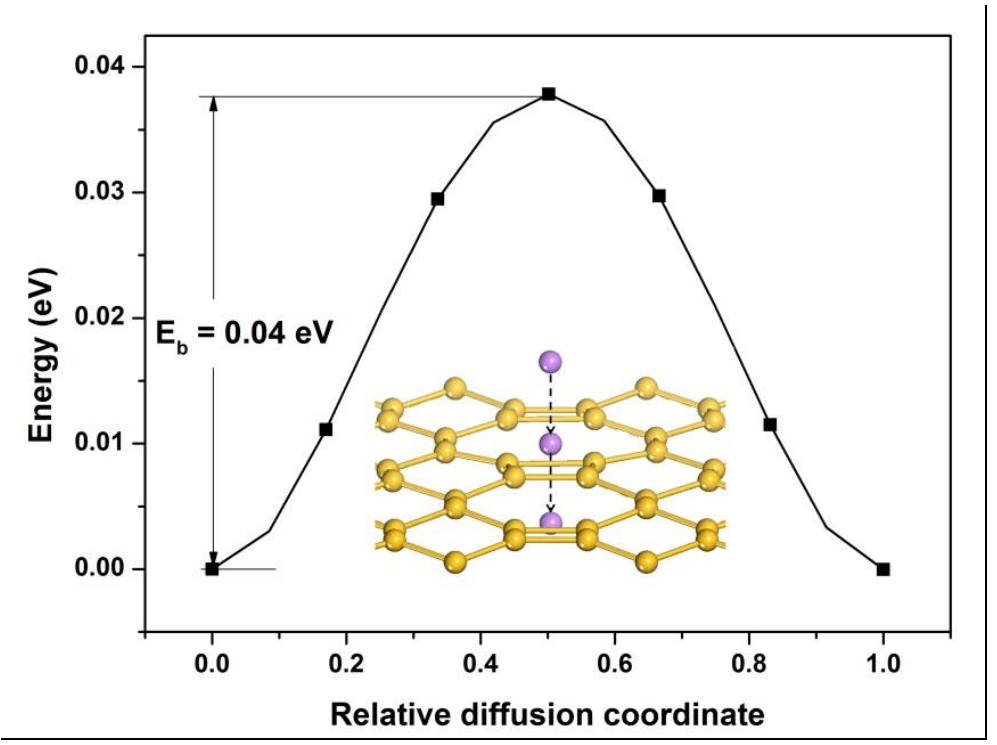

FIGURE 10 Energy curves for Li diffusion in the direction perpendicular to GB(4-8).

(a)

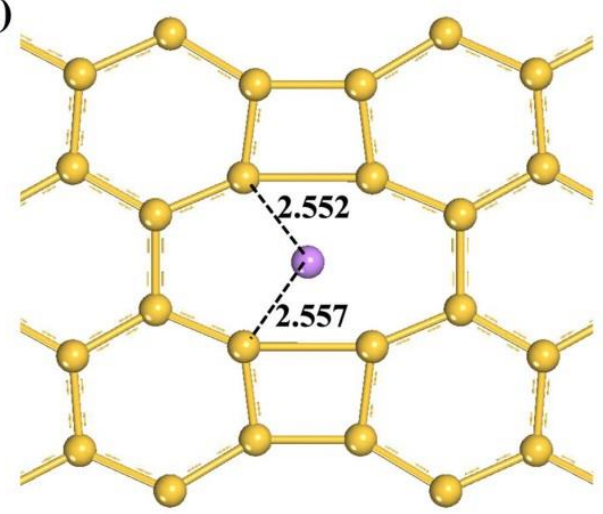

(b)

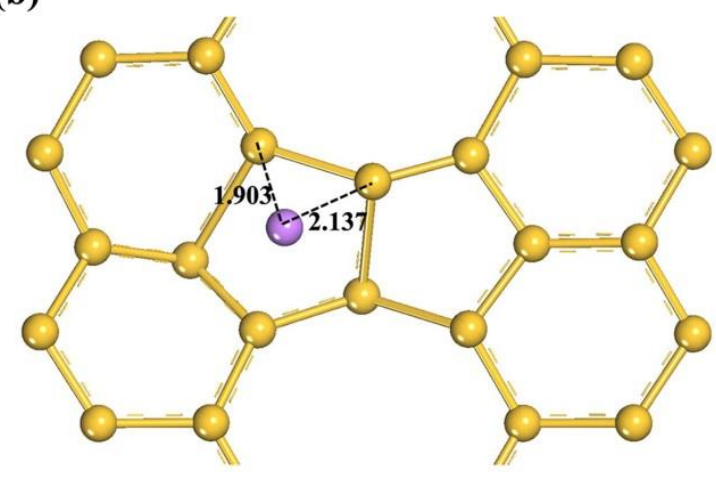

FIGURE 11 The bond lengths (in $\AA$ ) of Li through silicene (a) with GB(4-8) and (b) with (5-8) at the barrier states. 


\section{CONCLUSIONS}

In summary, based on density functional theory calculations, we have investigated adsorption and diffusion behavior of lithium atoms on silicene with $4 \mid 8$ and $5|5| 8$ grain boundaries. Our results show that $\mathrm{Li}$ is more strongly bounded to silicene with GBs compared with perfect ones. The donation of Li 2 s electron to the defects causes a strong interaction, thus enhances the adsorption. Moreover, diffusion of lithium atoms across and through the substrate is also an important consideration in the cycle performance of LIBs. It is found both GBs cases could significantly facilitate the diffusion behavior of the $\mathrm{Li}$ adatoms. Furthermore, the small energy barrier of $\mathrm{Li}$ atom enables rather easy penetration of the Li through the sheet of $\mathrm{GB}(4-8)$, suggesting the usages of the defective silicene as the sieve for Li atoms during the charge and discharge processes of LIBs. Due to these important features of the GB defective silicene, the present work is hoped to enlighten experimental realizations of the novel anodes for LIBs, and applications for the future energy resources eventually.

\section{AUTHOR CONTRIBUTIONS}

The work was conceptualized, investigated and originally drafted by X. Wang., under a general supervision by M. Zhang. X. Wang and W. Cao curated the data interpretation with helps from H. Z. Liu and M. Huttula. Project administrations were performed by Y. H. Luo and M. Huttula. All authors reviewed, edited, and approved 
the manuscript.

\section{FUNDING INFORMATION}

National Science Foundation of China, Grant/Award Number: 21303054;

Fundamental Research Funds for the Central Universities, Grant/Award Numbers: 222201714050, 222201714018; Profile Funding of Academy of Finland, Grant/Award Number: 311934.

\section{RESEARCH RESOURCES}

All the computation simulation was undertaken with the resources provided from the High Performance Computing Center of East China University of Science and Technology.

\section{Keywords:}

Lithium adsorption and diffusion, first-principles simulations, silicene, grain boundary

\section{REFERENCES}

[1] G. L. Soloveichik, Annu. Rev. Chem. Biomol. Enq. 2011, 2, 503.

[2] J. Liu, Adv. Funct. Mater. 2013, 23, 924. 
[3] H. Pauna, X. Y. Shi, M. Huttula, E. Kokkonen, T. H. Li, Y. H. Luo, J. Lappalainen, M. Zhang, W. Cao, Appl. Phys. Lett. 2017, 111, 103901.

[4] D. Pan, S. Wang, B. Zhao, M. Wu, H. Zhang, Y. Wang, Z. Jiao, Chem. Mater. 2009, $21,3136$.

[5] T. Bhardwaj, A. Antic, B. Pavan, V. Barone, B. D. Fahlman, J. Am. Chem. Soc. 2010, $132,12556$.

[6] J. R. Dahn, T. Zheng, Y. H. Liu, J. S. Xue, Science 1995, 270, 590.

[7] C. H. Sun, D. J. Searles, J. Phys. Chem. C 2012, 116, 26222.

[8] Y. Li, D. Wu, Z. Zhou, C. R. Cabrera, Z. Chen, J. Phys. Chem. Lett. 2012, 3, 2221.

[9] W. W. Lei, S. Qin, D. Liu, D. Portehault, Z. W. Liu, Y. Chen, Chem. Commun. 2013, 49, 352 .

[10] B. Feng, Z. J. Ding, S. Meng, Y. G. Yao, X. Y. He, P. Cheng, L. Chen, K. H. Wu, Nano Lett. 2012, 12, 3507.

[11] P. Vogt, P. De Padova, C. Quaresima, J. Avila, E. Frantzeskakis, M. C. Asensio, A. Resta, B. Ealet, G. Le Lay, Phys. Rev. Lett. 2012, 108, 155501.

[12] L. Meng, Y. Wang, L. Zhang, S. Du, R. Wu, L. Li, Y. Zhang, G. Li, H. Zhou, W. A. Hofer, H.-J. Gao, Nano Lett. 2013, 13, 685.

[13] A. Fleurence, R. Friedlein, T. Ozaki, H. Kawai, Y. Wang, Y. Yamada-Takamura, Phys. Rev. Lett. 2012, 108, 245501.

[14] C. K. Chan, R. N. Patel, M. J. O'Connell, B. A. Korgel, Y. Cui, ACS Nano 2010, $4,1443$.

[15] C. K. Chan, H. Peng, G. Liu, K. McIlwrath, X.F. Zhang, R.A. Huggins, Y. Cui, 
Nat. Nanotechnol. 2008, 3, 31.

[16] X. H. Liu, L. Zhong, S. Huang, S. X. Mao, T. Zhu, J. Y. Huang, ACS Nano 2012, $6,1522$.

[17] G. A. Tritsaris, E. Kaxiras, S. Meng, E. Wang, Nano Lett. 2013, 13, 2258.

[18] S. M. Seyed-Talebi, I. Kazeminezhada, J. Beheshtian, Phys. Chem. Chem. Phys. 2015, 17, 29689.

[19] X. F. Fan, W. T. Zheng, J. L. Kuo, ACS Appl. Mater. Interfaces 2012, 4, 2432.

[20] C. Uthaisar, V. Barone, Nano Lett. 2010, 10, 2838.

[21] X. Wang, Y. H. Luo, T. Yan, W. Cao, M. Zhang, Phys. Chem. Chem. Phys. 2017, $19,6563$.

[22] X. Y. Shi, Z. J. Huang, M. Huttula, T. H. Li, S. Y. Li, X. Wang, Y. H. Luo, M. Zhang, W. Cao, Crystals 2018, 8, 24.

[23] X. Y. Shi, S. Posysaev, M. Huttula, V. Pankratov, J. Hoszowska, J. C. Dousse, F. Zeeshan, Y. R. Niu, A. Zakharov, T. H. Li, O. Miroshnichenko, M. Zhang, X. Wang, Z. J. Huang, S. Saukko, D. L. González, S. van Dijken, M. Alatalo, W. Cao, Small 2018, 14, 1704526.

[24] D. Chiappe, C. Grazianetti, G. Tallarida, M. Fanciulli, A. Molle, Adv. Mater. 2012, 24, 5088 .

[25] H. Shu, D. Cao, P. Liang, X. Wang, X. Chen, W. Lu, Phys. Chem. Chem. Phys. 2014, 16, 304 .

[26] H. Sahin, J. Sivek, S. Li, B. Partoens, F. M. Peeters, Phys. Rev. B 2013, 88, 045434. 
[27] J. F. Gao, J. F. Zhang, H. S. Liu, Q. F. Zhang, J. J. Zhao, Nanoscale 2013, 5, 9785.

[28] O. Cretu, Y. -C. Lin, K. Suenaga, Nano Lett. 2014, 14, 1064.

[29] S. Najmaei, Z. Liu, W. Zhou, X. Zou, G. Shi, S. Lei, B. I. Yakobson, J. -C. Idrobo, P. M. Ajayan, J. Lou, Nat. Mater. 2013, 12, 754.

[30] O. V. Yazyev, S. G. Louie, Phys. Rev. B 2010, 81, 195420.

[31] Z. Song, V. Artyukhov, J. Wu, B. I. Yakobson, Z. Xu, ACS Nano 2015, 9, 401.

[32] Y. Liu, X. Zou, B. I. Yakobson, ACS Nano 2012, 6, 7053.

[33] A. N. Enyashin, M. Bar-Sadan, L. Houben, G. Seifert, J. Phys. Chem. C 2013, $117,10842$.

[34] H. Jamgotchain, Y. Colignon, B. Ealet, B. Parditka, J. Y. Hoarau, C. Girardeaux, B. Aufray, J. P. Bibérian, J. Phys.: Conf. Ser. 2014, 491, 012001.

[35] B. Yang, J. A. Boscoboinik, X. Yu, S. Shaikhutdinov, H. J. Freund, Nano Lett. 2013, 13, 4422 .

[36] L. J. Zhou, Z. F. Hou, L. M. Wu, Y. F. Zhang, J. Phys. Chem. C 2014, 118, 28055.

[37] X. L. Sun, Z. G. Wang, Y. Q. Fu, Carbon 2017, 116, 415.

[38] B. Delley, J. Chem. Phys. 1990, 92, 508.

[39] B. Delley, J. Chem. Phys. 1991, 94, 7245.

[40] B. Delley, Int. J. Quantum Chem. 1998, 69, 423.

[41] J. P. Perdew, K. Burke, M. Ernzerhof, Phys. Rev. Lett. 1996, 77, 3865.

[42] A. Tkatchenko, M. Scheffler, Phys. Rev. Lett. 2009, 102, 073005. 
[43] H. J. Monkhorst, J. D. Pack, Phys. Rev. B 1976, 13, 5188.

[44] T. A. Halgren, W. N. Lipscomb, Chem. Phys. Lett. 1977, 49, 225.

[45] G. Henkelman, H. Jonsson, J. Chem. Phys. 2000, 113, 9978.

[46] S. Li, Y. F. Wu, Y. Tu, Y. H. Wang, T. Jiang, W. Liu, Y. H. Zhao, Sci. Rep. 2014, 5, 7881.

[47] T. B. Douglas, L. F. Epstein, J. L. Dever, W. H. Howland, J. Am. Chem. Soc. 1955, 77, 2144.

[48] L. Pastewka, S. Malola, M. Moseler, P. Koskinen, J. Power Sources 2013, 239, 321.

[49] W. Cao, V. Pankratov, M. Huttula, X. Y. Shi, S. Saukko, Z. J. Huang, M. Zhang, Mat. Chem. Phys. 2015, 158, 89.

[50] M. Zhang, Z. J. Huang, X. Wang, H. Y. Zhang, T. H. Li, Z. L. Wu, Y. H. Luo, W. Cao, Sci. Rep. 2016, 6, 19504.

[51] J. Vähäkangas, P. Lantto, J. Vaara, M. Huttula, W. Cao, Chem. Commun. 2017, $53,5428$.

[52] F. Yao, F. Guenes, H. Q. Ta, S. M. Lee, S. J. Chae, K. Y. Sheem, C. S. Cojocaru, S. S. Xie, Y. H. Lee, J. Am. Chem. Soc. 2012, 134, 8646. 


\section{GRAPHICAL ABSTRACT}

First-principles calculations investigated lithium adsorption and diffusion behaviors on the silicene with $5|5| 8$ and $4 \mid 8$ grain boundaries (GBs). Besides increasing lithium adsorption energies, GBs in the $4 \mid 8$ case facilitate lithium migrations on the defective plane and crossing the monolayer. Yet, silicene with GBs are predicted as a superior anode material in future lithium ion batteries with high capacity and fast charging speed.

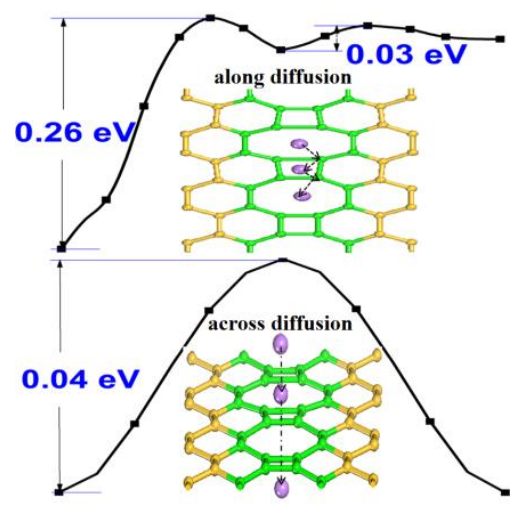

